\title{
A SPICE COMPATIBLE MODEL OF PERMANENT MAGNET DC MOTOR DRIVES
}

\author{
K.T. Chau ${ }^{\dagger}$ and C.C. Chan ${ }^{*}$ \\ ${ }^{\dagger}$ Department of Electrical Engineering \\ Hong Kong Polytechnic, Hong Kong \\ ₹ Department of Electrical and Electronic Engineering \\ The University of Hong Kong, Hong Kong
}

\begin{abstract}
A SPICE compatible model for the simulation of permanent magnet (PM) DC motor drives is presented. The key of the proposed model is to take into account of the armature reaction and magnet demagnetization, hence the shift of operating point at load conditions can be considered. Making use of this model, PSpice simulation of a typical PM DC motor drive is performed. Apart from the steady-state simulation, transient behaviours of the PM DC motor drive, including start-up, sudden load change and sudden reference change conditions, have been simulated conveniently. Based on a low-cost circuit simulator - PSpice, the developed model is especially helpful for undergraduate students.
\end{abstract}

\section{INTRODUCTION}

With the advent of high-energy permanent magnets (PMs), PM DC motor drives are becoming attractive in many industrial applications such as traction and servo control. The use of PMs for the field excitation of DC motors possesses definite advantages of higher power density, higher efficiency, lower electromechanical time constant, improved heat dissipation, less assembly problems and increased reliability [1], [2]. The conventional computer model for a PM DC motor is considered as a resistor, an inductor and a back emf connected in series. The induced back emf is proportional to the motor speed. This model is simple but inadequate to investigate both steady state and transient characteristics of a practical PM DC motor. One of the major reasons of this problem is due to the armature reaction effect and temporary magnet demagnetization.

Some papers have proposed better models for PM DC motors. However, one of the major disadvantages of those approaches is that these mathematical models cannot be easily linked to those of the power converters and controllers. On the other hand, some approaches are based on the finite element analysis [3], [4], which possess high accuracy but suffer from extremely high computational cost.

SPICE, its PC-version PSpice and its compatible simulators have been widely accepted and commonly used for electronic circuit simulation. Recently, many contributors have proposed models for the simulation of power converters, allowing the designers to perform the analysis in SPICE without any difficulty [5]. However, the use of SPICE for the simulation of DC motor drives is surprisingly little [6]. Even though, to the authors' knowledge, the simulation of PM DC motor drives using SPICE is almost zero. One major reason is due to the fact that there is a lack of available SPICE models for various components of the PM DC motor drive - PM DC motor, power converter, mechanical load, controller and sensor.

The purpose of this paper is to develop a SPICE compatible model for the simulation of PM DC motor drives. Hence, by using a low-cost circuit simulator such as PSpice [7], the undergraduate students can study both steady-state and transient performances of the PM DC motor drive in only one simulation. Without requiring tedious programming and avoiding the use of complex mathematics, the design work becomes more straight-forward and practical.

In this paper, the formulation of the SPICE compatible model for the PM DC motor drive is given in Section II. The corresponding PSPice simulation is presented in Section III which consists of various characteristics of the motor drive with open-loop and closed-loop control. The simulation is verified by comparing with the quoted data in a reference.

\section{SPICE COMPATIBLE MODEL}

The modelling of the PM DC motor drive is in modular structure. Each functional component of the motor drive is described by an individual SPICE compatible sub-model and is self-contained. This modular approach facilitates the model flexibility, capability and readability because a particular submodel can readily be used for other applications and its modification can be carried out in a convenient way. 
The functional components of a PM DC motor drive include the PM motor, mechanical load, DC chopper, controller and error detector. Because of the diversity of PMs, the PM motor component is further divided into the PM and armature circuit.

\section{A. Permanent Magnet Model}

The characteristic of PM materials is governed by its demagnetization curve. Its operating point is found at the intersection of the working line with the demagnetization curve. This working line depends on the geometry of the magnetic circuit. For exemplification, the mmf equation of a 2-pole PM DC motor [8], as shown in Fig. 1, is expressed as

$$
\frac{2 T N I_{c}}{r_{f}}+2 H_{m} L_{m}+2 H_{g} L_{s}=0
$$

where $r_{f}$ is the reluctance factor for the reluctance associated with materials used for the armature teeth and stator housing, $N I_{c}$ is the mmf over armature teeth 1 and $6, T$ is the number of armature teeth enclosed by one coil, $H_{m} L_{m}$ and $H_{g} L_{g}$ are respectively the mmf produced by the PM and mmf across the airgap. Substituting $H_{g}$ by $B_{g} / \mu_{o}$ and assuming $B_{g}$ equal to $B_{m}$, (1) can be rewritten as

$$
B_{m}=-\frac{\mu_{o} L_{m}}{L_{g}}\left(H_{m}+\frac{T N I_{c}}{L_{m} \boldsymbol{r}_{f}}\right)
$$

which is the expression to determine the working line. It should be noted that the operating point depends on the armature current. As shown in Fig. 2, the operating point is shifted downwards when the motor is at load conditions. It is therefore important to use PMs with high coercive forces to prevent from permanent demagnetization due to armature reaction.

By using the current-controlled voltage source in SPICE, the flux density can be related with the armature current. Hence, the SPICE compatible model of the PM can be obtained as shown in Fig. 3. The second stage is to convert the flux density to airgap flux by multiplying the area of the PM. Due to the difference in the axial length of the armature and the length of the PM as well as the armature slotting effect, the effective airgap flux is depicted by the third stage of the model. Since the demagnetization curve is generally nonlinear, it is represented by using a lookup table in SPICE format.

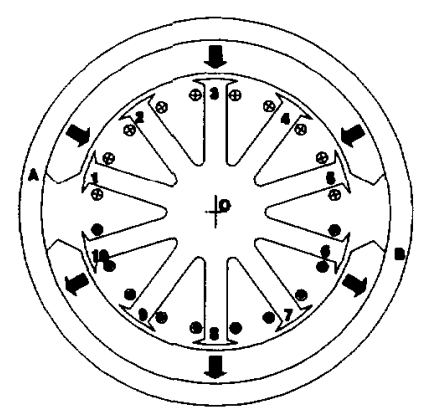

Fig. 1. Geometry of a 2-pole PM DC motor.

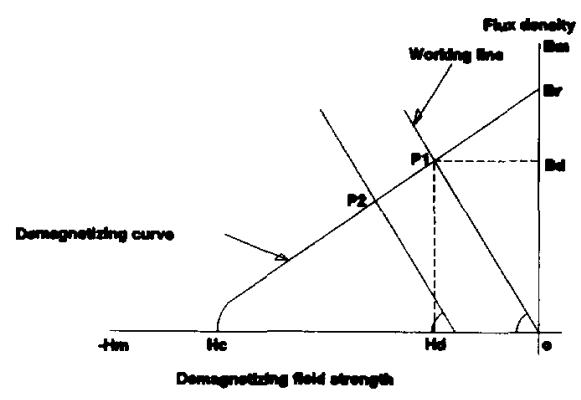

Fig. 2. Shifting of operating point at load conditions.

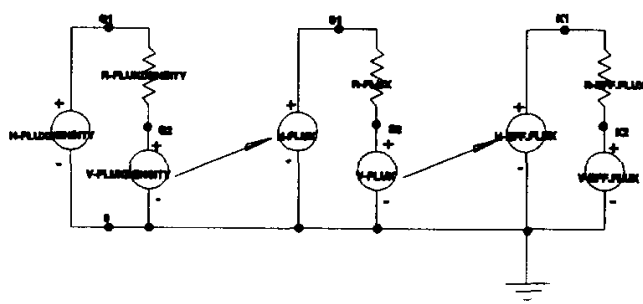

Fig. 3. PM model. 


\section{B. Armature Circuit Model}

The SPICE compatible model for the armature circuit of the PM DC motor is shown in Fig. 4, where $R_{a}$ and $L_{a}$ are respectively the series resistance and inductance of the armature winding. The dependent voltage source $H-B A C K$ is the induced back emf proportional to the effective airgap flux and motor speed. Another voltage source $V-A . C U R$ is dummy which is used for sensing the value of armature current in the circuit.

Since there is no field control for the PM DC motor, the torque control is generally based on the control of armature current. Therefore, there is no magnetic saturation when overload torque is needed. Nevertheless, the magnetic saturation effect can be handled in SPICE by piecewisely linearizing the magnetic saturation curve into lines with different slopes.

\section{Mechanical Load Model}

By using the similarity between mechanical and electrical systems, the SPICE compatible model for the mechanical load is shown in Fig. 5, where the inductor represents the load inertia, the resistor represents the frictional loss and the current flow in the circuit is equivalent to the load speed of the mechanical system. The voltage sources $H-D E V . T Q$ and $V-M . L O A D$ are the electromagnetic torque and load torque, respectively. Sometimes, when the mechanical load system involves a significant damping torque such as liquid cooling, an equivalent capacitor can be added in series.

\section{DC Chopper Model}

The DC chopper can readily be modelled by using the voltage controlled switch available in SPICE. The on/off of the switch is dictated by comparing the carrier and output feedback signals. Although this modelling is straight-forward, it suffers from a serious problem that the required simulation time is extremely tedious. The reason is due to the fact that the internal time step during simulation is limited by the switching period while hundreds of switching cycles are required to reach the steady state. Recently, a systematic approach to modelling the switching part in switchmode power supplies has been proposed [9], [10]. The basic idea is to separate the switching part of the converter circuit into a minimum separable switching configuration (MISSCO) which can be represented by an identifiable equivalent circuit.

By applying the MISSCO approach to the DC chopper, the transistor and diode of the DC chopper are extracted and then replaced by an equivalent circuit consisting of a dependent current source, a dependent voltage source and a parasitic resistance that is a function of duty cycle. As shown in Fig. 6, this equivalent model is already in SPICE compatible format and can readily be merged with the armature circuit of the motor.

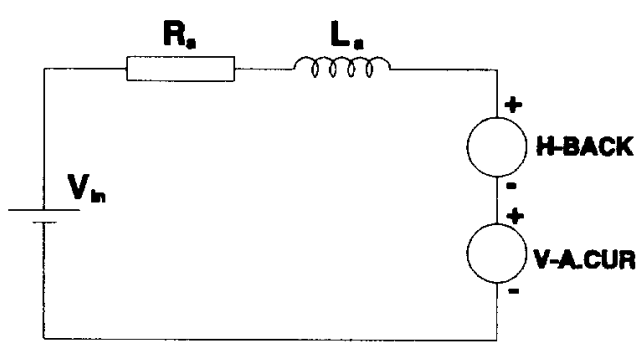

Fig. 4. Armature circuit model.

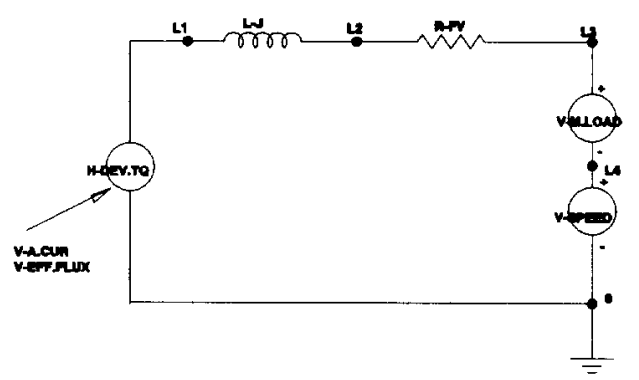

Fig. 5. Mechanical load model.

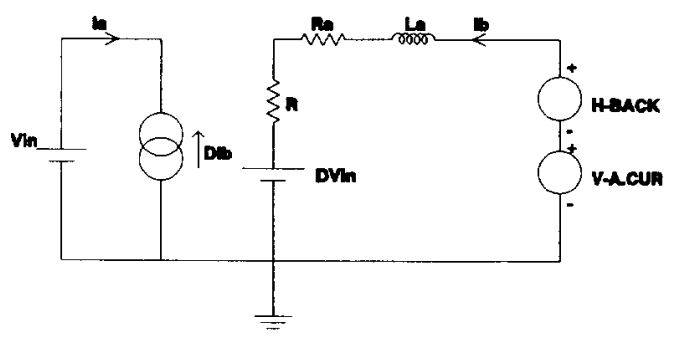

Fig. 6. DC chopper model. 


\section{E. Error Detector Model}

In the mechanical load model, the speed is represented by the current flow in the circuit. In order to obtain the speed feedback for the controller, a current controlled voltage source is used to copy the speed signal. Another voltage source is used to represent the reference speed. The voltage difference of these voltage sources represents the speed error. The model of this error detector is shown in Fig. 7.

\section{F. Controller Model}

Since the controller of this motor drive is an analogue electronic circuit, it is particularly suitable for directly modelling using the built-in libraries in SPICE. Since PID control is the most popular control strategy in industrial applications, the PID controller model is formed as shown in Fig. 8. By adjusting the values of $R B, C B, R F$ and $C F$, the transient characteristics such as the rising time, settling time and maximum overshoot can be fine tuned.

\section{PSPICE SIMULATION}

Both open-loop and closed-loop simulations are carried out in which the former describes the start-up transient characteristics in the absence of the DC chopper, error detector and controller while the latter deals with the transient characteristics during starting and under sudden load change. The practical data for the simulation is given in Table $\mathrm{I}$.

\section{A. Open-Loop Simulation}

Fig. 9 shows the start-up transient characteristic of the motor speed. It can be found that the rising time is $3.4 \mathrm{~s}$, the settling time is $4.8 \mathrm{~s}$ and there is no overshoot because of the large mechanical inertia. With the load torque of $0.115 \mathrm{Nm}$, the steady-state speed is equal to $207 \mathrm{rad} / \mathrm{s}$.

Figs. 10 and 11 show the start-up transient characteristic of the armature current. It can be found that the maximum overshoot is $365 \%$, the rising time is only $252 \mu \mathrm{s}$ and the settling time is $5.8 \mathrm{~s}$. The high start-up armature current is due to the low back emf during low speeds. The great difference between the rising and settling times is due to the great difference between the electrical and mechanical time constants. Similarly, Figs. 12 and 13 show the start-up transient characteristic of the effective airgap flux. It is found that the effective flux changes from $0.778 \mathrm{mWb}$, with the minimum of $0.373 \mathrm{mWb}$, to $0.667 \mathrm{mWb}$, depending on the value of armature current. It verifies that the operating point of the PM is shifted downwards due to armature reaction.

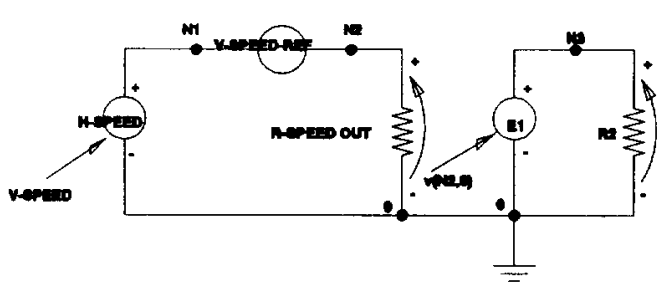

Fig. 7. Error detector model.

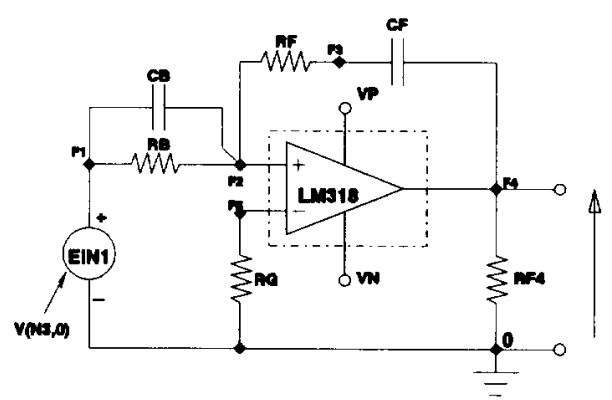

Fig. 8. Controller model.

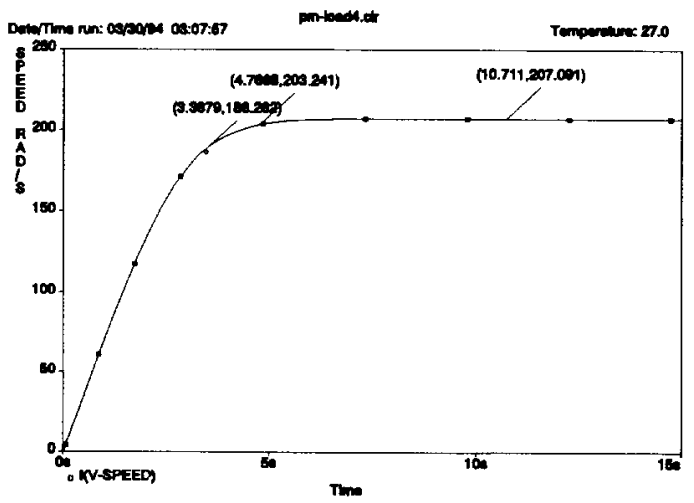

Fig. 9. Open-loop speed response. 


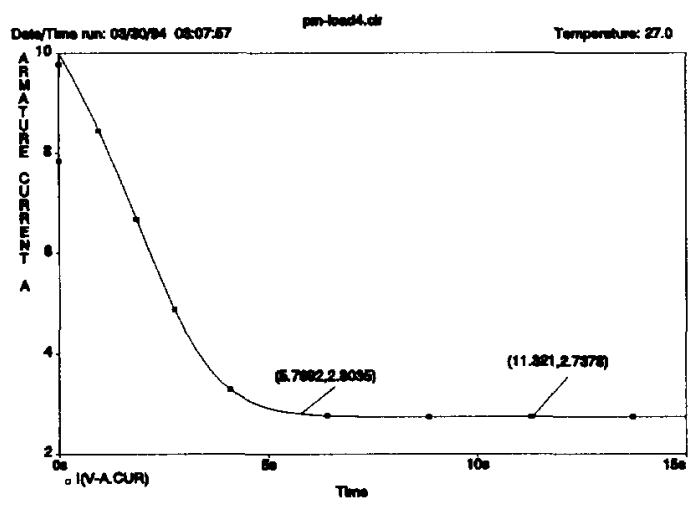

Fig. 10. Open-loop armature current response.

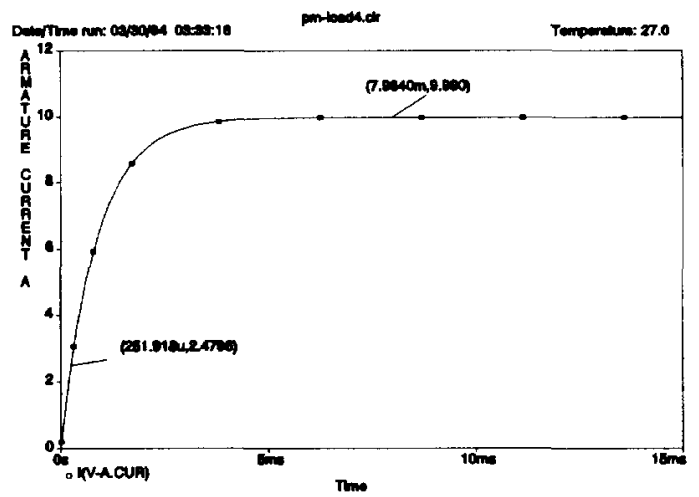

Fig. 11. Enlarged armature current response.

\section{B. Verification}

Since the technical parameters for the above simulation are based on a practical PM DC motor described in [8], the simulation results are verified by comparing them with those quoted data. It can be found that the maximum discrepancy between them is less than $2 \%$.

TABLE I

\section{PARAMETERS OF THE PM DC MOTOR}

Ceramic ferrite $\mathrm{Ba}-\mathrm{Sr}$

$B_{r}=0.38 \mathrm{~T}, \mathrm{H}_{\mathrm{c}}=200 \mathrm{kA} / \mathrm{m}$

$12 \mathrm{~V}, 2.75 \mathrm{~A}, 210 \mathrm{rad} / \mathrm{s}$

$\mathrm{R}_{\mathrm{a}}=12 \mathrm{\Omega}, \mathrm{L}_{\mathrm{a}}=1.06 \mathrm{mH}$

$\mathrm{J}=0.0017 \mathrm{kgm}, \mathrm{k}=0.041 \mathrm{~V} / \mathrm{rad} / \mathrm{s}$

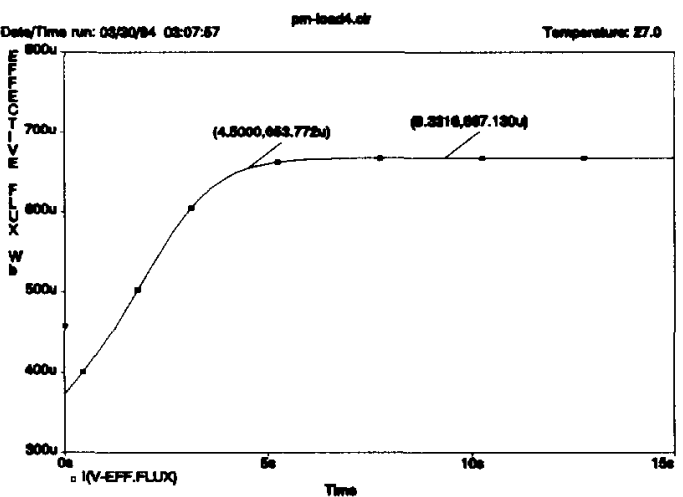

Fig. 12. Open-loop effective flux response.

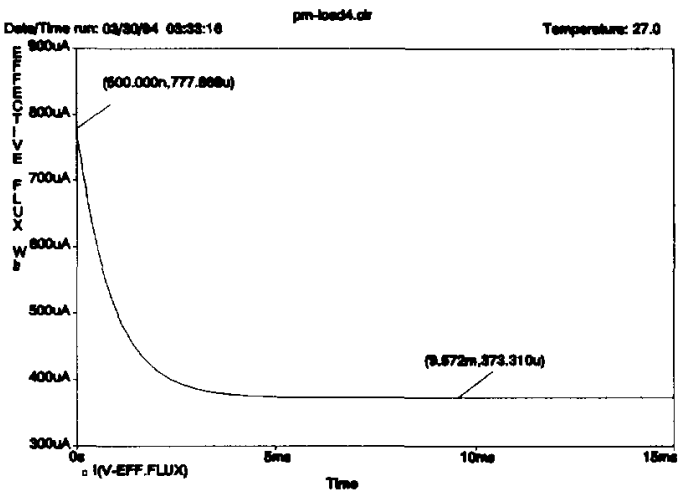

Fig. 13. Enlarged effective flux response.

\section{Closed-Loop Simulation}

In order to have a more realistic simulation, the transient characteristics of the PM DC motor drive are simulated. Figs 14, 15 and 16 illustrate the transient characteristics of motor speed, effective airgap flux and developed torque, respectively when the load is changed suddenly. It can be found that the resulting characteristics are as expected - the motor speed is regulated to follow the desired value, the effective flux is reduced by the armature reaction field and the developed torque is increased to balance the new load. Moreover, it should be noted that the PID controller is so tuned that a critical damping occurs and there is no overshoot. Due to the flexibility of the proposed model, other transient characteristics such as due to the sudden reference change can readily be simulated. 


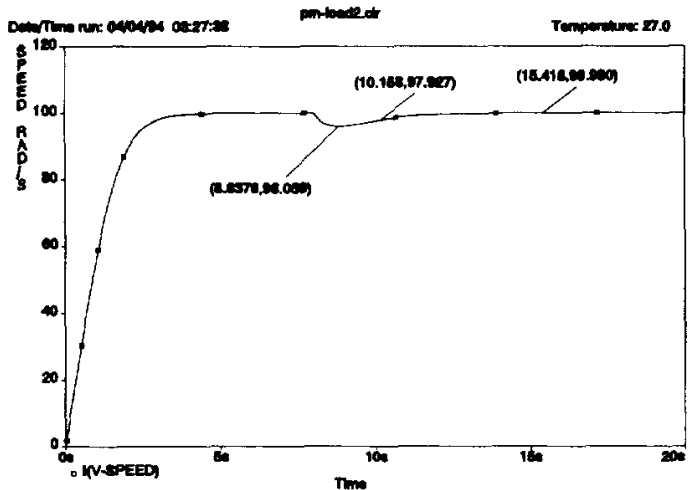

Fig. 14. Closed-loop speed response.

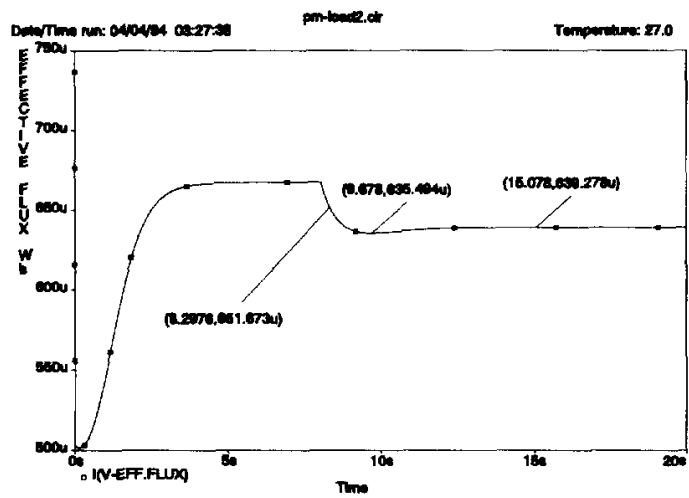

Fig. 15. Closed-loop effective flux response.

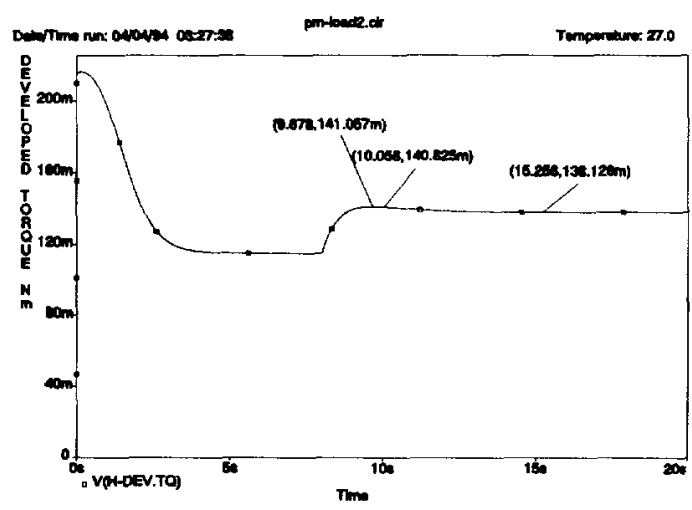

Fig. 16. Closed-loop torque response.

\section{CONCLUSION}

A complete set of SPICE compatible models for the simulation of PM DC motor drives has been developed. By taking into account the motor armature reaction and temporary magnet demagnetization effects, these models are simple, accurate and easy to use. By employing the MISSCO approach to ignore the switching details of the DC chopper, the simulation time can be greatly reduced. Open-loop and closedloop simulations based on the developed models are carried out to investigate both steady-state and transient performances of a PM DC motor drive.

\section{ACKNOWLEDGMENT}

The authors gratefully acknowledge the contribution of Mr. W.K. Chan, and the support in part of Hong Kong Polytechnic.

\section{REFERENCES}

[1] T.J.E. Miller, Brushless Permanent-Magnet and Reluctance Motor Drives. Oxford Univ. Press, 1989.

[2] K.T. Chau, "Computer-aided design of a permanent magnet motor," Elect. Machines Power Syst., vol. 19, no. 4, pp. 501-511, 1991.

[3] S.J. Salon, "Finite element analysis of electric machinery," IEEE Comput. Appl. Power Mag., pp. 29-32, 1990.

[4] C.C. Chan and K.T. Chau, "Finite element method based electrical machine design using distributed computing, " Computers Ind., vol. 17, no. 4, pp. 367-374, 1991.

[5] M.H. Rashid, SPICE for Power Electronics and Electric Power. Prentice Hall, 1993.

[6] Y.C. Liang and V.J. Gosbell, "DC machine models for SPICE2 simulation," IEEE Trans. Power Electron., vol. 5, no. 1, pp. 16-20, 1990.

[7] PSpice Circuit Analysis. MicroSim Corp. 1992.

[8] M.G. Say and E.O. Taylor, Direct Current Machines. Pitman, 1980.

[9] K.T. Chau, Y.S. Lee and A. Ioinovici, "Computer-aided modeling of quasi-resonant converters in the presence of parasitic losses by using the MISSCO concept," IEEE Trans. Ind. Electron., vol. 38, no. 6, pp. 455-462, 1991.

[10] Y.S. Lee, Computer-Aided Analysis and Design of Switch-Mode Power Supplies. Marcel Dekker, 1993. 